\title{
5 Os transtornos típicos de cultura e a enfermagem transcultural: running amok
}

\author{
Patrícia Sofia Caldeira Mota ${ }^{1}$; Francisco Miguel Correia Sampaio
}

\section{RESUMO}

\section{Enquadramento}

A Running Amok é um Transtorno Típico de Cultura (CBS) cujos maiores níveis de incidência e prevalência se verificam em países como a Malásia, bem como em outros países do Sudeste Asiático. A ocorrência de casos semelhantes tem, no entanto, vindo a ser verificada, igualmente, nas sociedades mais industrializadas. Nesse sentido, importa perceber a pertinência da Enfermagem Transcultural como perspectiva de actuação perante as CBS e, em particular, perante a Running Amok.

\section{Metodologia}

A metodologia adoptada assentou, essencialmente, na revisão bibliográfica realizada, sobretudo, a partir da consulta de artigos científicos relativos à temática das $\mathrm{CBS}$ e da Psiquiatria Cultural e Etnopsiquiatria.

\section{Resultados}

A Running Amok pode ser dividida em quatro fases: a primeira fase é caracterizada por um período de depressão e irritabilidade; na segunda fase a pessoa manifesta um impulso agressivo incontrolável; na terceira fase a pessoa apresenta um comportamento homicida; na quarta fase a pessoa experiencia um período de amnésia parcial, isto se, sobreviver ao estádio anterior. A Enfermagem Transcultural surgiu com o objectivo de promover a prestação de cuidados de Enfermagem adaptados aos diferentes contextos culturais das pessoas, pelo que se verifica que, na actualidade, a competência cultural é encarada como um elemento-chave na prestação de cuidados de saúde.

\section{Conclusões}

Considerando a hipótese de integração de algumas CBS, comoaRunning Amok, no novo DSM-V, torna-seindispensável o estudo deste tipo de patologias, bem como a aquisição de competências culturais, por parte dos enfermeiros, para lidar com esse tipo de questões podendo, para tal, ser utilizadas como base as noções ligadas à Enfermagem Transcultural.

\section{PALAVRAS-CHAVE: Running Amok; Transtorno Típico de Cultura; Psiquiatria Cultural; Enfermagem Transcultural.}

\begin{abstract}
\section{Background}

Running Amok is a Culture-Bound Syndrome (CBS); its most relevant incidence and prevalence rates are identified in countries like Malaysia, as well as in other countries of Southeast Asia. However, the occurrence of similar cases has also been verified in industrial societies. Considering this evidence, it is imperative to understand Transcultural Nursing importance as an intervention strategy before CBS and, particularly, before Running Amok.
\end{abstract}

\section{Methods}

The adopted methodology was literature review, having been consulted, mainly, scientific articles related to specific themes: CBS, Cultural Psychiatry and Ethnopsychiatry.

\section{Results}

Running Amok can be divided into four stages: in the first stage, the patient is irritable and depressed; in the second stage, the patient is uncontrollably aggressive; in the third stage, the patient presents with homicidal behaviour; in the fourth stage, the patient experiences a partial amnesia period, if he/she survives to the previous stage. Transcultural Nursing emerged with the objective of promoting Nursing care adapted to different cultural contexts. So, nowadays, cultural competence is understood as a key element to formal caregiving.

\section{Conclusions}

Considering the probable integration of some CBS, as Running Amok, in DSM-V, it is undeniable that nurses should study this kind of pathologies and acquire cultural competencies, in order to cope with this kind of situation. To do so, nurses can use, in the baseline, concepts related to Transcultural Nursing.

KEYWORDS: Running Amok; Culture-Bound Syndrome; Cultural Psychiatry; Transcultural Nursing

${ }^{1}$ Licenciada em Enfermagem - ESEP / Mestranda em Enfermagem de Saúde Mental e Psiquiatria, patricia_mota0912@hotmail.com

2 Enfermeiro, Hospital de Braga - Serviço de Internamento de Psiquiatria / Mestrando em Enfermagem de Saúde Mental e Psiquiatria, fmcsampaio@gmail.com Submetido em 17-09-2011. Aceite em 16-12-2011.

Citação: Mota, P. S. C.; Sampaio, M. C. (2011). Os transtornos típicos de cultura e a enfermagem transcultural: running amok. Revista Portuguesa de Enfermagem de Saúde Mental, 6, 28-35. 


\section{INTRODUÇÃO}

A Etnopsiquiatria surge, na actualidade, como um importante componente da Enfermagem de Saúde Mental e Psiquiatria, considerando o mundo global em que se desenvolvem as práticas de prestação de cuidados de saúde. Nesse contexto, surge a necessidade, por parte dos enfermeiros, de dominar cada vez melhor as competências relacionadas com a Enfermagem Transcultural.

A Running Amok, ou simplesmente Amok, é um dos Transtornos Típicos de Culturas (CBS) mais frequentes na actualidade, sobretudo em países como a Malásia, estando classificada na revisão da quarta edição do Diagnostic and Statistical Manual of Mental Disorders ${ }^{\circledR}$ (DSM-IV-TR $®$ ) (American Psychiatric Association, 2002).

O presente artigo visa analisar algumas questões ligadas à Psiquiatria Cultural e à Etnopsiquiatria, e em particular à Running Amok. Nesse sentido, serão abordados os seguintes aspectos: definição, etiopatogenia, incidência e prevalência, critérios de diagnóstico, sintomatologia, tratamento, prognóstico e intervenções de Enfermagem associadas à Running Amok. Por fim, será realizada uma análise criticoreflexiva sobre o trabalho desenvolvido.

\section{METODOLOGIA}

O artigo a elaborar será construído, sob o ponto de vista metodológico, com base na revisão bibliográfica realizada, predominantemente, a partir de livros presentes na biblioteca da Escola Superior de Enfermagem do Porto, de artigos indexados na base de dados EBSCOhost ${ }^{\circledR}$, PubMed $\AA$, MEDLINE ${ }^{\circledR}$ e de outros artigos presentes em sítios da Internet dedicados à divulgação de trabalhos científicos.

\section{PSIQUIATRIA CULTURAL E ETNOPSIQUIATRIA}

A cultura influencia aspectos fundamentais da identidade e funcionamento social do Homem, afectando a forma como este expressa o seu sofrimento e experiencia a patologia. A cultura, define ainda o que é normal e desviante. A Psiquiatria Cultural incentiva o profissional de saúde a compreender o cliente segundo uma perspectiva cultural, e não numa perspectiva meramente biomédica (Bhugra, Kalra, 2010). Ao longo dos sub-capítulos seguintes serão explorados os conceitos de Psiquiatria Cultural e de Etnopsiquiatria, bem como os seus desafios futuros, sendo ainda realizada uma abordagem à Enfermagem Transcultural.

\section{Conceitos e desafios}

A doença mental manifesta-se de formas diferentes nas sociedades sendo que estas, por sua vez, têm também formas distintas de lidar com ela. Nas sociedades mais ocidentais, prevalece o controlo institucional da doença, bem como a atribuição da responsabilidade para resolver o problema ao profissional de saúde. No entanto, existem sociedades que não fazem uso das instituições para controlar a doença, nem atribuem demasiada responsabilidade aos profissionais. Nessas mesmas sociedades, não são utilizados os argumentos científicos para trabalhar com o doente, dando-se preferência ao conhecimento tradicional, que é compartilhado por todos. Assim, o "psiquiatra tradicional" faz uso desse mesmo conhecimento e do apoio de toda a comunidade para exercer a sua função (Noronha, 2004).

No que concerne à Psiquiatria Cultural, este termo foi introduzido por Kraeplin em dois artigos de reflexão acerca das suas experiências com doentes mentais, em Java, no início do Século XX (Kraeplin, 1904, cit. por Stompe, Wintrob, 2007). Durante as décadas que se seguiram aos relatos pioneiros de Kraeplin, o papel emergente da Psiquiatria Cultural foi fortalecido por um crescente corpo de investigação sistemática que conduziu à percepção mais profunda das características colectivas e individuais dos seres humanos. Na actualidade, é comum a partilha de conhecimento específico de cada sociedade relativamente a questões como a religião, política, educação ou economia, mas também acerca de sistemas simbólicos e semânticas que apresentam influências directas ou indirectas na fenomenologia e nos aspectos relatados acerca de perturbações psiquiátricas (Stompe, Wintrob, 2007).

Os rápidos progressos científicos da Psiquiatria Cultural no Século XX foram, em grande parte, despoletados pelos enormes desafios sócio-políticos e éticos que surgiram na sequência das guerras, resultando em fortes vagas de migração, forçada e voluntária, com a consequente readaptação aos novos ambientes. Essa readaptação levou, necessariamente, a um crescendo de situações de stress por aculturação, conflitos intergeracionais de valores, distress psíquico e sintomatologia psiquiátrica (Stompe, Wintrob, 2007).

Todos esses momentos de desenvolvimento histórico do século passado levaram à promoção da diferenciação da Psiquiatria Cultural em três áreas importantes: a Psiquiatria Transcultural/Comparativa; a Etnopsiquiatria; e a Psiquiatria de Migração. (Stompe, Wintrob, 2007).

A Etnopsiquiatria é, assim, o ramo da Psiquiatria Cultural mais próximo da etnologia e da ciência etnográfica, descrevendo a formação de perturbações mentais específicas de culturas. Constituem um exemplo, as CBS ou o impacto específico de padrões sócio-culturais e religiosos, bem como as atitudes terapêuticas a adoptar perante o doente mental. (Kleinman, 1988, cit. por Stompe, Wintrob, 2007). 
O propósito da Etnopsiquiatria é o de estabelecer uma linguagem que possa ser compreendida por todos, valorizar o meio onde reside a pessoa doente, e utilizar-se esse mesmo meio como factor de recuperação. Assim, no trabalho etnopsiquiátrico, a participação do doente e dos familiares no tratamento é activa, com o objectivo último de entender a etiologia do problema e de procurar soluções para o caso (Noronha, 1986, cit. por Noronha, 2004). Neste sentido, a Etnopsiquiatria não pretende ser mais do que uma subdivisão de uma especialidade, mas com a função particular de chamar a atenção para os componentes socioculturais em torno do doente mental que, ao longo dos tempos, tem vindo a ser subjugado por uma visão redutora do seu problema sofrendo, por isso, sérias consequências (Collomb, 1975, cit. por Noronha, 2004).

A Etnopsiquiatria foi construída a partir de um princípio metodológico extremamente relevante neste contexto: a complementaridade não é uma teoria, mas antes uma generalização metodológica; a complementaridade não exclui nenhum método, todos os métodos são considerados válidos (Devereux, 1972, cit. por Baubet, Moro, 2000). É no âmbito da ligação verificada entre a fragilidade e o contexto, que a Etnopsiquiatria adquiriu a razão da sua existência. Por entre as suas diversas redes e filiações, esta apresenta um triplo sentido no centro dos seus discursos e práticas: primeiramente, enfatiza a dignidade de todas as culturas como uma plataforma a partir da qual os diagnósticos podem ser realizados; em segundo lugar, traz ao de cima o facto de o encarceramento não ser a melhor terapia para as perturbações mentais; finalmente, tem em linha de conta o contexto e o ambiente como aspectos proeminentes de qualquer narrativa realizada pelo doente acerca de si mesmo, e pelo terapeuta acerca da doença (Bidima, 2000).

\section{As culturas e a prática de enfermagem}

A Enfermagem Transcultural nasceu na década de 1950 com o objectivo de promover uma prestação de cuidados congruente com as culturas, proporcionando assim um cuidar em Enfermagem mais humanístico, seguro e significativo para as pessoas de diversas culturas em todo o Mundo. Assim, a Enfermagem Transcultural defende que todas as culturas têm o direito de ter os seus valores, crenças e formas de vida reconhecidas, respeitadas e suportadas pela sua saúde e bem-estar (Leininger, 1997, Leininger, 2008). No mesmo sentido, tem vindo a ser defendida a importância da competência cultural na prestação de cuidados de saúde, na medida em que muitos erros de diagnóstico e de tratamento se relacionam directamente com factores como o desconhecimento cultural ou o défice ao nível da comunicação (Campinha-Bacote, Campinha-Bacote, 2009). A comunicação transcultural inclui factores que devem ser tidos em consideração quando os enfermeiros interagem com os clientes e com os membros da família de contextos culturais que diferem do seu (Andrews, 2003, cit. por MaierLorentz, 2008). Assim, questões simples, como o contacto ocular, devem ser extremamente cuidados, já que os enfermeiros portugueses são ensinados a manter o contacto ocular com o cliente quando, por exemplo, na cultura árabe, o contacto ocular directo é considerado indelicado, e até mesmo agressivo. Da mesma forma, os nativos norteamericanos consideram o contacto ocular directo como sendo algo impróprio; na sua cultura, sentar-se no chão durante o diálogo é sinónimo de estar a escutar atentamente a pessoa com quem se está a falar (Maier-Lorentz, 2008).

Nos países ocidentais os enfermeiros são ensinados a usar o toque como forma de comunicação terapêutica com os clientes (Understanding Transcultural Nursing, 2005, cit. por Maier-Lorentz, 2008), no entanto, algumas culturas proíbem ou restringem o toque a outras pessoas. Assim, é frequente, clientes que provêm de contextos árabes ou hispânicos não permitirem que os prestadores de cuidados do sexo masculino toquem algumas partes do corpo feminino. Para além disso, as mulheres de ambas as culturas podem também ser impedidas de cuidar de clientes do sexo masculino. Os asiáticos não aprovam o toque na cabeça porque acreditam que aí reside a fonte da força da pessoa (Maier-Lorentz, 2008).

O silêncio, nos países ocidentais, é vulgarmente interpretado de forma negativa, podendo os enfermeiros sentir-se desconfortáveis quando existe um período de silêncio durante o diálogo com o cliente. Porém, em algumas culturas o silêncio apresenta uma conotação extremamente positiva. Por exemplo, é comum nos nativos norte-americanos o uso do silêncio como forma de mostrar respeito pela pessoa que está a falar. O silêncio também é obrigatório quando se fala com os idosos de culturas asiáticas, já que é um sinal de grande respeito pelas pessoas mais velhas. Mesmo em populações próximas de Portugal, como na França e Espanha, as pessoas demonstram concordância com o uso do silêncio (Maier-Lorentz, 2008).

Existem ainda algumas particularidades ao nível do espaço e distância, bem como no que concerne às crenças em saúde. Assim, por exemplo, as pessoas de descendência norte-americana, geralmente, sentem-se mais confortáveis quando não estão em contacto próximo com outras pessoas. Por outro lado, os hispânicos e asiáticos tendem a sentir-se muito confortáveis quando estão próximos de outrem (Andrews, 2003, cit. por Maier-Lorentz, 2008). As pessoas que acreditam que têm controlo sobre os seus eventos de vida crêem ter controlo sobre o seu estado de saúde (exemplo dos asiáticos e americanos). Por outro lado, de um modo geral, os hispânicos sentem que têm menos controlo sobre as suas vidas e tendem a ser mais fatalistas na sua visão acerca da saúde, acabando por ser menos colaborantes no cumprimento de uma dieta prescrita ou regime medicamentoso (Maier-Lorentz, 2008).

A Enfermagem Transcultural é essencial na prática diária de Enfermagem. O crescente número de clientes de variados 
contextos culturais faz com que os enfermeiros tenham que adquirir conhecimentos transculturais, como forma de estarem mais sensibilizados para as necessidades dos clientes das mais diversas culturas, na medida em que as sociedades se têm vindo a tornar cada vez mais globais e complexas (Maier-Lorentz, 2008).

\section{CBS - TRANSTORNOS TÍPICOS DE CULTURAS}

No século XIX houve um aumento circunstancial de explorações médicas por todo o mundo e começaram a surgir diversos relatos médicos de transtornos que até então eram desconhecidos para os investigadores. Com o estudo destas patologias começou a inferir-se que os factores culturais representavam um papel fundamental no seu desenvolvimento. A primeira classificação destes fenómenos deve-se a Yap, em 1951, após a qual surgiram novas propostas de classificação que iremos abordar de seguida (Tseng, 2006).

\section{Conceito e breve historial}

Segundo Tseng (2006, p. 554), Transtornos Típicos de Culturas (CBS) são "condições mentais ou síndromes psiquiátricas cuja manifestação ou ocorrência estão relacionadas intimamente com factores culturais, garantindose assim que a sua avaliação e tratamento é feito segundo uma perspectiva cultural”.

Com o aumento das viagens pelo mundo, os relatos de distúrbios psiquiátricos associados a factores culturais foi aumentando (Gaw, Bernstein, 1992) e a classificação destes fenómenos teve vários estádios, de acordo com a própria evolução histórica da psiquiatria cultural (Tseng, 2006).

Numa fase inicial Yap (1951, cit. por Tseng, 2006) classificou estes fenómenos como "distúrbios psiquiátricos peculiares" que mais tarde vieram a apelidar-se de CBS (Yap, 1967, cit. por Tseng, 2006), classificação que se mantém até hoje. No entanto, têm vindo a surgir novas sugestões para a sua classificação, nomeadamente "culture-related specific conditions" (Tseng, McDermott, 1981, cit. por Tseng, 2006) e mais recentemente "culture-related specific syndromes" (Tseng, 2001, cit. por Tseng, 2006). Estas propostas surgem com o objectivo de haver uma maior precisão na definição destes fenómenos (Tseng, 2006).

O número de propostas que surgiram para a classificação destes fenómenos, é, na opinião de Hughes (1996, cit. por Pussetti, 2006) um indício de que a sua classificação sempre foi problemática, uma vez que foi criada para abranger fenómenos que por si só também eram difíceis de classificar.

\section{AMOK}

Uma das CBS mais comummente referenciadas na literatura (sobretudo internacional) é a Running Amok, um distúrbio presente na Malásia, que iremos explanar, seguidamente, de forma mais aprofundada. Assim, nos sub-capítulos seguintes serão abordadas, inicialmente, a definição e a etiopatogenia da Running Amok, os critérios de diagnóstico e sintomatologia da patologia e, finalmente, o tratamento e prognóstico da mesma.

\section{Definição e etiopatogenia}

Amok, também conhecida como running amok ou amuk, é um termo de origem malaia que significa "louco com uma raiva incontrolável", que acontece quase exclusivamente nos homens (Schmidt, Hill, Guthrie, 1977). Foi inicialmente identificada na Malásia, muito embora também tenham sido reportados episódios de amok noutras regiões do Sudeste Asiático, incluindo a Sumatra, a Papua Nova Guiné, Singapura, Indonésia, Filipinas e Laos (Gaw, Bernstein, 1992). Segundo Metzger (1887, cit. por Schmidt et al., 1977) os nativos da Malásia apresentavam uma predisposição para o assassínio colectivo, uma vez que, estavam sempre armados com um kris ou uma faca (a arma habitual dos episódios de amok), iniciando o ataque tendo por base algumas situações como ciúmes por uma mulher ou humilhação de algum género. Outras armas utilizadas nestes ataques eram a parang, machados, bastões, facas e armas (Schmidt et al., 1977).

Embora a expressão amok seja usada comummente, a sua principal utilização está ligada a um Transtorno Típico de Cultura, na cultura malaia. Segundo Martin (1999, p. 66) "este termo descreve um comportamento homicida e consequentemente suicida de indivíduos instáveis mentalmente que resulta em múltiplas lesões e mortes para os outros". Na linguagem malaia, mengamok refere-se ao acto de "ficar louco de raiva" (running amok) e o pengamok é a pessoa que protagoniza o acto (Gaw, Bernstein, 1992). Os indivíduos corriam selvaticamente, apresentando uma força e persistência extraordinárias, sendo normalmente assassinados ou contidos, mas quando tal não se verificava, o indivíduo apresentava um colapso abrupto sem razão aparente e já durante a recuperação afirmava que tinha estado mata gelap (com os olhos escurecidos), era então capturado e tornava-se escravo do rajah (Metzger, 1887, cit. por Schmidt et al., 1977).

Carr e Tan (1976, cit. por Gaw, Bernstein, 1992, p. 790) descreviam o amok na cultura malaia, como um "canal para expressar uns impulsos agressivos nas pessoas que normalmente estavam condicionadas a reprimir a sua raiva".

O amok foi evoluindo ao longo dos séculos: originalmente era o grito de guerra dos piratas malaios que tinham como objectivo saquear outros barcos e esta acção era bem aceite socialmente e considerada honrosa; do século 
XVI ao século XVIII o pengamok iniciava as suas acções de uma forma consciente e deliberada, como terrorismo político, mas evitando prejudicar outras pessoas que não os alvos seleccionados inicialmente; devido ao aumento da incidência destes casos, o governo colonial britânico, definiu, em 1893, que estes casos deveriam ser julgados em tribunal, tendo-se verificado um decréscimo significativo na sua incidência; no século XIX a natureza do comportamento sofreu uma alteração drástica, uma vez que os episódios de amok deixaram de ser premeditados e os homicídios em massa ocorriam num estado dissociativo e com amnésia posterior; já na segunda metade do século XIX a frequência de casos de amok diminuiu drasticamente e os indivíduos apresentavam um longo historial de distúrbios psicóticos, em vez de reacções dissociativas (Tseng, 2006).

A sua classificação enquanto CBS deve-se à descoberta desta patologia, há dois séculos atrás, em "tribos primitivas", acreditando-se que os factores culturais desempenhavam um papel predominante na sua patogenia, devido ao seu isolamento geográfico e crenças religiosas que levavam ao aparecimento de uma perturbação mental que não era observada no resto do mundo (Martin, 1999). No século XIX este síndrome era relatado com alguma frequência em alguns grupos étnicos da Malásia, nomeadamente os Bugis, os Javanese e os Malays (Gaw, Bernstein, 1992).

Westermeyer (1973, cit. por Gaw, Bernstein, 1992), um psiquiatra do Minnesota que se dedicou ao estudo dos episódios de amok, verificou a possível existência de um padrão epidémico nos casos de amok que avaliou na Malásia, na Tailândia, em Laos e nas Filipinas, acreditando que existia uma maior predisposição para este tipo de comportamento após a exposição a episódios de amok. A sua teoria apoiava-se em situações como o caso do "grenade-amok" que ocorreu em 1959 durante um festival religioso em Laos, após o qual se verificou um aumento gradual da incidência de casos de amok, atingindo 20 casos reportados em 1966. Também Schmidt et al. (1977) estudaram os casos de amok no Este da Malásia, sendo que dos 24 casos analisados, apenas um era referente a uma mulher, pelo que podemos inferir que é mais prevalente no sexo masculino.

Desde o aparecimento dos primeiros casos de amok que se tem vindo a especular sobre as causas deste transtorno (Gaw, Bernstein, 1992). Diversos autores apontaram como causas orgânicas a epilepsia (Zaquirre, 1957, cit. por Gaw, Bernstein, 1992, Kraepelin, 1904, cit. por Schmidt et al., 1977), infecções como a malária ou a sífilis (Kraepelin, 1904, cit. por Schmidt et al., 1977, Van Loon, 1928, cit. por Gaw, Bernstein, 1992), esquizofrenia (Kraepelin, 1904, cit. por Schmidt et al., 1977, Zaquirre, 1957, cit. por Gaw, Bernstein, 1992), depressão (Ellis, 1901, cit. por Gaw, Bernstein, 1992), psicoses (Weidman, 1971, cit. por Gaw, Bernstein, 1992) e reacções dissociativas (Esser, 1961, cit. por Gaw, Bernstein, 1992). Segundo os dados recolhidos no estudo de Schmidt et al. (1977), os autores acreditam que a amok pode aparecer associada a uma extensa variedade de distúrbios psicopatológicos.

No entanto, e uma vez que, a maioria dos casos observados não revelaram qualquer uma destas patologias, os investigadores têm enfatizado o papel da cultura na génese deste problema (Gaw, Bernstein, 1992). Westermeyer (1973, cit. por Gaw, Bernstein, 1992) definiu três variáveis culturais que estariam envolvidas no desenvolvimento deste fenómeno, entre elas: a susceptibilidade individual, uma experiência cultural que incluía uma consciencialização sobre a violência de amok e, por fim, rápidas alterações socioculturais.

Mais recentemente, Martin (1999, pp. 68-69) afirma que, tendo em conta a escassa literatura sobre amok, pode concluir-se que condições psiquiátricas, a personalidade, a patologia, e/ou perdas recentes, são factores importantes na sua patogénese, definindo como factores de risco:

"história de comportamento violento e/ou ameaças; tentativas de suicídio anteriores; stress interpessoal significativo; transtornos paranóides, anti-sociais, narcisistas, ou traços de personalidade borderline; história de psicose ou comportamento violento durante uma alteração de humor; distúrbios psicóticos com delírios persecutórios; distúrbios psicóticos com alucinações que incentivavam a violência; actividade delirante; problemas com o emprego".

Segundo Schmidt et al. (1977) podem classificar-se quatro fases da doença: a primeira fase é caracterizada por um período de depressão e irritabilidade com respostas agressivas do indivíduo quando alguém tenta penetrar no seu espaço de segurança; na segunda fase o indivíduo manifesta um impulso agressivo incontrolável; na terceira fase o indivíduo apresenta um comportamento homicida que se mantém até que o pengamok seja contido, morto ou entre num estado de exaustão e estupor; na quarta e última fase, os indivíduos experienciam um período de amnésia parcial, isto se, sobreviverem ao estádio anterior.

Nos dois últimos séculos tem-se verificado um decréscimo na incidência dos episódios de amok, acreditando-se que as influências da civilização ocidental e os intercâmbios culturais foram eliminando os factores culturais que se acreditava serem os principais responsáveis por este comportamento violento na Malásia e noutras cultura asiáticas (Martin, 1999). No entanto, tem-se verificado um aumento de ocorrência de casos semelhantes nas sociedades industrializadas, nomeadamente um caso reportado em 1987 na Grã-Bretanha (Kon, 1994), mas, uma vez que se acreditava profundamente que os factores culturais induziam o aparecimento de amok, não foi feita uma ligação com estes casos de violência em massa (Martin, 1999). Os media, as testemunhas e os relatórios policiais descrevem os atacantes como pessoas estranhas ou irritadas e impulsivas, sugerindo distúrbios da personalidade, distúrbios paranóides, ou ainda síndromes depressivos resultante de uma perda severa (Martin, 1999). Outra das semelhanças com os episódios de amok, 
é o número de vítimas resultantes, muito embora as armas utilizadas actualmente, como os revólveres e as espingardas, sejam bastante distintas das espadas malaias utilizadas há dois séculos atrás (Martin, 1999).

São conhecidas duas formas de amok, sendo que a forma mais comum, beramok, encontra-se associada a uma perda pessoal e é precedida por um período de humor depressivo; por seu lado, a amok, foi associada a um ataque a outrem, despoletado por raiva, insultos ou vingança. Assim sendo, e tendo por base os mais recentes casos de amok reportados, acredita-se que a beramok está relacionada com perturbações depressivas, enquanto que o amok parece estar associada a psicoses, distúrbios da personalidade ou delírios (Martin, 1999).

\section{Diagnóstico e sintomatologia}

Devido ao reporte, cada vez mais frequente, de distúrbios relacionados com factores culturais, como o koro, amok e pibloktoq, que chegaram a atingir as centenas em todo o mundo (Gaw, Bernstein, 1992), começou a inferir-se que alguns sistemas de valores, estruturas sociais e crenças partilhadas podiam despoletar formas psicopatológicas específicas restritas a determinadas áreas geográficas (Yap, 1969, cit. por Gaw, Bernstein, 1992). Assim sendo, o desenvolvimento na década de 90 , da décima edição da Internacional Classification of Disease (ICD) e da quarta edição do Diagnostic and Statistical Manual of Mental Disorders (DSM) acabou por se tornar a oportunidade ideal "para uma análise e classificação mais sofisticada destes distúrbios e para realizar estudos comparativos em várias culturas" (Gaw, Bernstein, 1992, p. 789).

Com o aumento das viagens pelo mundo e da emigração, podia verificar-se um aumento da ocorrência destes fenómenos nos países industrializados, pelo que se tornava pertinente dar uma maior atenção aos distúrbios relacionados com os factores culturais na DSM, muito embora a sua terceira edição fosse utilizada essencialmente nas culturas ocidentais (Gaw, Bernstein, 1992).

O processo de introdução do amok no DSM-IV passou por diversas fases, entre elas: a análise do amok e a discussão dos seus factores culturais específicos; a estipulação de critérios que definissem um distúrbio como "especificamente relacionado com os factores culturais" tendo por base um paradigma proposto previamente para a classificação destes distúrbios; e por último, a argumentação para que seja feita a classificação do amok no DSM-IV (Gaw, Bernstein, 1992).

"Um acto de amok é a combinação de um distúrbio do humor, do comportamento e da cognição" (Gaw, Bernstein, 1992, p. 791). O distúrbio de humor não é específico e normalmente só se verifica em retrospectiva, a amnésia posterior também demonstra alguma inconsistência, até porque alguns dos observadores eram da opinião de que os indivíduos simulavam a amnésia para saírem impunes, pelo que o aspecto mais importante a considerar seria a alteração dos comportamentos verificada ao cometer os homicídios (Gaw, Bernstein, 1992). Estas conclusões levaram a que os autores sugerissem, numa fase inicial, que o amok fosse classificado como um distúrbio de controlo de impulsos, mas, segundo Simons e Hughes (1992, cit. por Gaw, Bernstein, 1992) deveria ser classificada como um distúrbio explosivo isolado, uma vez que este se caracteriza por um único episódio de perda do controlo de impulsos que conduz a um acto violento, com impacto catastrófico noutros indivíduos, e cuja informação disponível, não justifica o diagnóstico de esquizofrenia.

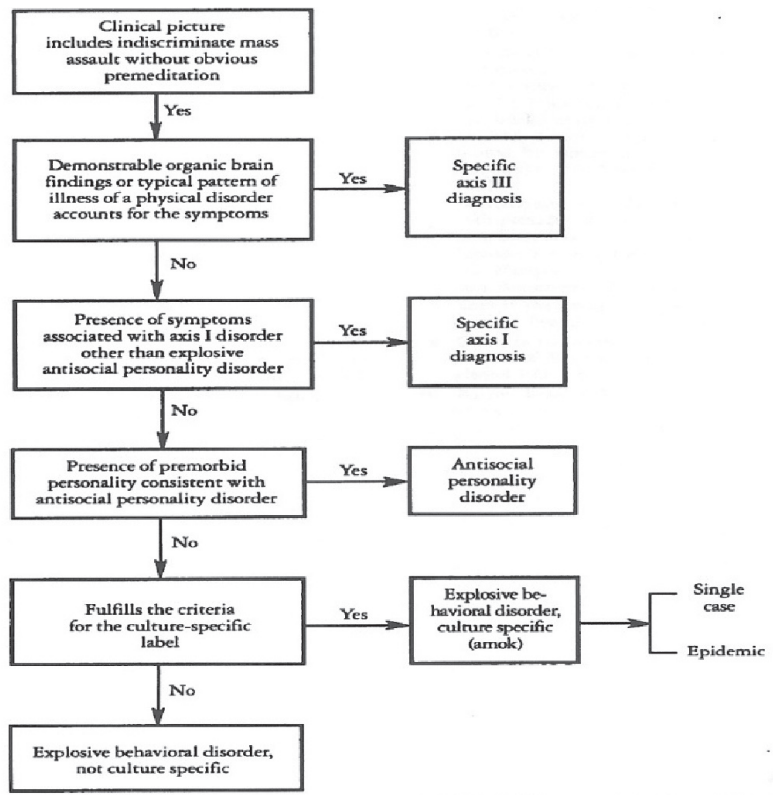

Alguns investigadores propuseram a criação de um apêndice que incluísse os distúrbios relacionados com os factores culturais, no entanto Gaw e Bernstein (1992), refutaram esta opção, uma vez que acreditavam que esta estratégia levaria a que se inferisse que a cultura era o componente mais importante destas síndromes; no entanto, muitos destes "distúrbios culturais" apresentam características semelhantes aos distúrbios ocidentais. Assim sendo, a resolução deste dilema, passou pela revisão da categoria de "distúrbios explosivos intermitentes" que era contemplada no DSM-III-R, passando a existir no DSM-IV, a categoria de "distúrbios comportamentais explosivos" e criada uma árvore de decisão (Figura 1) (Gaw, Bernstein, 1992).

\section{Tratamento e prognóstico}

Martin (1999), afirma que o amok deverá ser considerado como um possível resultado de uma psicose ou de uma severa perturbação da personalidade que esteja subdiagnosticada ou não tratada e, uma vez que é praticamente impossível parar um ataque de amok, torna-se imperioso prevenir que este aconteça. 
A primeira abordagem de intervenção passa pela identificação de indivíduos que apresentem condições psiquiátricas ou stressores psicossociais que predisponham para a ocorrência de casos de running amok (Martin, 1999). O autor identifica várias características comuns aos indivíduos que desenvolveram este distúrbio, que poderão contribuir para a identificação dos indivíduos predispostos, sendo elas:

"distúrbios depressivos psicóticos, ou distúrbios de humor, especialmente distúrbios bipolares; distúrbios da personalidade, com surtos de violência assim como distúrbios antissociais e personalidades borderline; distúrbios de personalidade paranóide e/ou alucinações com temas persecutórios e comportamentos agressivos como defesa a ameaças identificadas; perdas pessoais significativas e stressores psicossociais; comportamento suicida e homicida e pensamentos agressivos, sem esperança e vingativos; distúrbios psicóticos com delírios persecutórios sobre o próprio; esquizofrenia paranóide com alucinações de comando ou de actos violentos, que o individuo tende a obedecer." (Martin, 1999, p. 68).

A segunda abordagem de intervenção passa pela identificação das situações clínicas sub-diagnosticadas e subsequente tratamento farmacológico e não farmacológico das mesmas, para que não se venha a verificar um episódio de amok (Martin, 1999).

A intervenção de Enfermagem deve ocorrer numa perspectiva de prevenção (sobretudo primária) da ocorrência de episódios de amok, em indivíduos que apresentem um dos distúrbios mentais prévios que, aparentemente, conferem uma maior predisposição para desenvolver este tipo de patologia, bem como uma herança cultural que se coaduna com a mesma. Assim sendo, a intervenção deve compreender, essencialmente, dois níveis: o coping, na medida em que é importante que o doente seja capaz de desenvolver mecanismos de adaptação a situações limite que poderão despoletar o episódio de amok; e na gestão de comportamentos, e de controlo de agressividade (Dochterman, Bulechek, 2008). Estas abordagens, que visam o controlo de eventos que se pensam favorecer um episódio de amok, como os sentimentos de raiva, a resposta face a insultos ou vingança que referimos anteriormente, podem então diminuir a probabilidade da sua ocorrência. Assim, a intervenção ao nível dos comportamentos deve ser mais dirigida aos indivíduos que, ainda antes de desenvolverem qualquer episódio de running amok, já apresentam sinais evidentes de dificuldades ao nível da assertividade e do controlo dos impulsos e da agressividade.

Mediante a perturbação de base, e sobretudo no caso de o indivíduo apresentar pensamentos disfuncionais, crenças erróneasouerros cognitivos, deverecorrer-seàreestruturação cognitiva, ou seja, intervir no âmbito da terapia cognitiva. No caso de a pessoa apresentar problemas ao nível cognitivo e comportamental deve, preferencialmente, intervir-se aos dois níveis, em simultâneo. Porém, no caso de tal não ser possível, deve dar-se preferência, numa fase inicial, à abordagem cognitiva, passando-se então para a abordagem comportamental. Apenas no caso de o indivíduo apresentar comportamentos que, dada a sua gravidade, coloquem em risco a sua própria vida ou a vida de outrem, deve optar-se, primeiramente, por uma abordagem comportamental, dada a necessidade imperiosa de modificar o comportamento, mesmo antes de trabalhar com o utente no sentido de implementar um plano de cuidados que se centre no ponto de vista cognitivo.

\section{CONSIDERAÇÕES FINAIS}

Em suma, é importante que o enfermeiro tenha em conta a herança cultural do doente a quem está a prestar cuidados, de forma a não desvalorizar os valores e crenças da sua cultura, e, desta forma, poder demonstrar respeito pela pessoa e prestar cuidados de saúde diferenciados e mais humanizados.

No que diz respeito ao Amok, consegue perceber-se que existem algumas incongruências, nomeadamente no que concerne aos factores de risco, uma vez que englobam os distúrbios psiquiátricos mais comuns nas culturas ocidentais, o que dificulta a possibilidade de prevenção da ocorrência de um episódio de Amok, já que este poderia ocorrer na grande maioria dos doentes; por outro lado, a inclusão dos factores culturais como uma das causas precipitantes destes episódios restritos a determinadas áreas geográficas, acaba por não explicar o porquê da ocorrência de casos de Running Amok na actualidade, em sociedades ocidentais, como por exemplo o caso identificado na Grã-Bretanha em 1987, anteriormente referido, no qual não se verificavam características culturais semelhantes às existentes nas tribos malaias e os diversos casos de violência em massa reportados todos os anos, que, muito embora apresentem características muito semelhantes às encontradas aos casos de Amok, não tiveram a mesma classificação devido à inexistência de factores culturais evidentes que pudessem ter despoletado o episódio.

A inclusão desta patologia no DSM-IV acaba por ser um avanço significativo, na medida em que a existência de uma árvore de decisão fornece critérios específicos para o seu diagnóstico. No entanto a inclusão dos factores culturais como um dos critérios para o seu diagnóstico exclui uma grande parte dos possíveis casos relatados nas últimas décadas.

Assim sendo, seria pertinente uma avaliação mais aprofundada dos factores de risco de Amok, para ser possível estabelecer correctamente um plano de cuidados diferenciado e que fosse eficaz na prevenção deste distúrbio. 


\section{REFERÊNCIAS BIBLIOGRÁFICAS}

APA - American Psychiatric Association (2002). Manual de Diagnósticos e Estatística das Perturbações Mentais (DSMIV-TR) (4 ${ }^{\mathrm{a}}$ Ed.). Lisboa: Climepsi.

Baubet, T., Moro, M. R. (2000). "L'Approche Ethnopsychiatrique". Enfances \& Psy, (12), pp. 111-117. Consultado em 25 de Abril de 2011. Disponível em:

http://www.cairn.info/revue-enfances-et-psy-2000-4-page-111.htm.

Bidima, J. (2000). "Ethnopsychiatry and its Reverses: Telling the Fragility of the Other". Diogenes, 48 (1), pp. 68-82. Consultado em 25 de Abril de 2011. Disponível em: http:// web.ebscohost.com/ehost/pdfviewer/pdfviewer?vid=4\&hid= $18 \&$ sid $=d b 7 f a f 44-9 d b b-487 e-85 d 4-99 d 4 b 887 d 56 f \% 40$ sessi onmgr12.

Bhugra, D., Kalra, G. (2010). "Cross Cultural Psychiatry: Context and Issues". Journal of Pakistan Psychiatric Society, 7 (2), pp. 51-54. Consultado em 13 de Maio de 2011. Disponível em: http://web.ebscohost.com/ehost/pdfviewer/ pdfviewer? vid=6\&hid=11\&sid=72ab8dd2-a84e-4cd2-b61d2e00615ce905\%40sessionmgr13.

Campinha-Bacote, A., Campinha-Bacote, J. (2009). "Extending a Model of Cultural Competence in Health Care Delivery to the Field of Health Care Law". Journal of Nursing Law, 13 (2), pp. 36-44. Consultado em 13 de Maio de 2011. Disponível em: http://web.ebscohost.com/ehost/pdfviewer/ pdfviewer?vid=9\&hid=25\&sid=72ab8dd2-a84e-4cd2-b61d2e00615ce905\%40sessionmgr13.

Dochterman, J. M, \& Bulechek, G. M. (2008). Classificação das Intervenções de Enfermagem (NIC) (4 ${ }^{\mathrm{a}}$ Ed.). Porto Alegre: Artmed.

Gaw, A., Bernstein, R. (1992). "Classification of Amok in DSM-IV". Hospital \& Community Psychiatry, 43 (8), pp. 78993. Consultado em 28 de Abril de 2011. Disponível em: http:// ps.psychiatryonline.org/cgi/reprint/43/8/789.

Kon, Y. (1994). "Amok". The British Journal of Psychiatry: The Journal of Mental Science, 165 (5), pp. 685-89. Consultado em 28 de Abril de 2011. Disponível em: http://bjp.rcpsych. org/cgi/reprint/165/5/685.

Leininger, M. (1997). "Future Directions in Transcultural Nursing in the 21st Century". International Nursing Review, 44, (1), pp. 19-23. Consultado em 13 de Maio de 2011. Disponível em: http://web.ebscohost.com/ehost/pdfviewer/p dfviewer?vid=12\&hid=123\&sid=72ab8dd2-a84e-4cd2-b61d2e00615ce905\%40sessionmgr13.
Leininger, M. (2008). "Foreword". Contemporary Nurse: A Journal for the Australian Nursing Profession, 28 (1/2), pp. 35. Consultado em 13 de Maio de 2011. Disponível em: http:// web.ebscohost.com/ehost/pdfviewer/pdfviewer?vid=17\&hid $=15 \&$ sid $=72$ ab8dd2-a84e-4cd2-b61d-2e00615ce905\%40se ssionmgr13.

Maier-Lorentz, M. M. (2008). "Transcultural Nursing: Its Importance in Nursing Practice". Journal of Cultural Diversity, 15 (1), pp. 37-43. Consultado em 25 de Abril de 2011. Disponível em: http://web.ebscohost.com/ehost/pdfviewer/ pdfviewer ? vid=9\&hid=7\&sid=db7faf44 $-9 \mathrm{dbb}-487 \mathrm{e}-85 \mathrm{~d} 4$ 99d4b887d56f\%40sessionmgr12.

Martin, M. (1999). "Running Amok: A Modern Perspective on a Culture-Bound Syndrome". Primary Care Companion to the Journal of Clinical Psychiatry, 1 (3), pp. 66-70. Consultado em 19 de Abril de 2011. Disponível em: http://www.ncbi.nlm. nih.gov/pmc/articles/PMC181064/pdf/i1523-5998-001-030066.pdf

Noronha, M. (2004). "A Etnopsiquiatria na Sociedade Contemporânea". Revista Presença, 8 (28). Consultado em 25 de Abril de 2011. Disponível em: http://www.revistapresenca. unir.br/artigos_presenca/28marcosdenoronha_aetnopsiquia trianasociedade.pdf.

Schmidt, K., Hill, L., Guthrie, G. (1977). "Running Amok”. The International Journal Of Social Psychiatry, 23(4), pp. 264-74. Consultado em 28 de Abril de 2011. Disponível em: http://isp. sagepub.com/content/23/4/264.full.pdf+html.

Stompe, T., Wintrob, R. (2007). "Cultural Psychiatry: Perspectives for the Next Decade". Official Journal of World Association of Cultural Psychiatry, 2 (2/3), pp. 44-46. Consultado em 25 de Abril de 2011. Disponível em: http:// www.wcprr.org/pdf/02-23/2007.0203.4446.pdf.

Tseng, W. (2006). "From Peculiar Psychiatric Disorders through Culture-bound Syndromes to Culture-related Specific Syndromes". Transcultural Psychiatry, 43 (4), pp. 554-576. Consultado em 16 de Abril de 2011. Disponível em: http://tps. sagepub.com/content/43/4/554.full.pdf+html. 